\title{
SOME PROPERTIES OF SIMILAR PAIRS
}

\author{
GUNNAR BLOM,* University of Lund \\ LARS HOLST, ${ }^{* *}$ Royal Institute of Technology, Stockholm
}

\begin{abstract}
In a given set, the elements are compared pairwise. The number $W$ of similar pairs is studied, that is, the number of pairs with a certain property in common. Under certain conditions, $W$ has, approximately, a Poisson distribution. Examples are considered connected with the birthday problem and with a circle problem involving DNA breakages.
\end{abstract}

BIRTHDAY PROBLEM; DISSOCIATED STATISTICS; DNA BREAKAGES; MULTINOMIAL DISTRIBUTION; POISSON APPROXIMATION

\section{Introduction and summary}

Let $\left\{A_{1}, A_{2}, \cdots, A_{n}\right\}$ be a set of elements. The elements $A_{i}$ and $A_{j}$ are said to form.a similar pair if they are related in a given way; more briefly, they are then said to be similar. For example, the $A$ 's may be coloured balls which are called similar if they have the same colour.

Introduce the indicator random variables $I_{i j}$, where $I_{i j}=1$ if $A_{i}$ and $A_{j}$ are similar, and $I_{i j}=0$ otherwise. We are interested in the total number of similar pairs

$$
W=\sum_{i<j} I_{i j}
$$

The sum consists of $M=\left(\begin{array}{l}n \\ 2\end{array}\right)$ terms.

The elements are assumed to be generated by some chance mechanism. We shall consider the following situation:

(a) The indicator random variables $I_{i j}$ have common mean $p$.

(b) If the random variables $I_{i j}$ and $I_{k l}$ have no indices in common, they are independent.

(c) If $I_{i j}$ and $I_{k l}$ have exactly one index in common then $\operatorname{Cov}\left(I_{i j}, I_{k l}\right)=c$.

Hence the random variables $I_{i j}$ are dissociated; cf. Barbour and Eagleson (1984).

Let $X$ be a random variable assuming integer values $0,1, \cdots$. Set

$$
\Delta_{X}=\frac{\operatorname{Var}(X)}{E(X)}-1
$$

This quantity can be positive, negative or zero. If $\Delta_{X}$ is near zero, $X$ can often be approximated by a Poisson distribution. Such cases will be encountered in this paper.

In Section 2, we derive the mean and variance of $W$ and prove our main theorem which concerns the variational distance between $W$ and a Poisson random variable $Z$ with the same mean as $W$. In Section 3, examples are given concerning the uniform distribution and the multinomial distribution. As special cases we consider a birthday problem and a problem concerning DNA breakages.

Received 20 March 1989; revision received 30 June 1989.

* Postal address: Department of Mathematical Statistics, University of Lund, Box 118, S-221 00 Lund, Sweden.

** Postal address: Department of Mathematics, Royal Institute of Technology, S-100 44 Stockholm, Sweden. 


\section{Properties of $W$}

It follows from the assumptions (a)-(c) that the mean and variance of $W$ are given by

$$
E(W)=M p ; \quad \operatorname{Var}(W)=M p(1-p)+2 M(n-2) c .
$$

Hence we obtain

$$
\Delta_{W}=2(n-2) c / p-p
$$

Consider the variational distance

$$
d(W, Z)=\sup _{B}|P(W \in B)-P(Z \in B)|
$$

between $W$ and $Z$, where $Z \sim$ Poisson $(M p)$.

Theorem 1 . The variational distance satisfies the inequality

$$
d(W, Z)<\left(1-e^{-E(W)}\right)\left(\Delta_{W}+4 n p\right) .
$$

Proof. Consider a sum $W$ of dissociated Bernoulli random variables $I_{i j}, 1 \leqq i<j \leqq n$, identically distributed or not. Set $p_{i j}=P\left(I_{i j}=1\right)$. According to Theorem 1 in Barbour and Eagleson (1984) and Lemma 4 in Barbour and Eagleson (1983) we have

$$
d(W, Z) \leqq \frac{1-e^{-E(W)}}{E(W)}\left[\sum p_{i j}^{2}+\sum^{\prime} p_{i j} p_{k l}+\sum^{\prime} E\left(I_{i j} I_{k l}\right)\right]
$$

Here $\Sigma$ denotes summation over $1 \leqq i<j \leqq n$ and $\Sigma^{\prime}$ summation over all pairs of indices $(i, j),(k, l)$ with exactly one index in common.

Since

$$
\begin{aligned}
E(W) & =\sum p_{i j} \\
\operatorname{Var}(W) & =\sum p_{i j}\left(1-p_{i j}\right)+\sum^{\prime} E\left(I_{i j} I_{k l}\right)-\sum^{\prime} p_{i j} p_{k l}
\end{aligned}
$$

we can rewrite the right-hand side of the inequality in the form

$$
\left(1-e^{-E(W)}\right)\left[\Delta_{W}+R\right] \text {, }
$$

where

$$
R=2\left(\sum p_{i j}^{2}+\sum^{\prime} p_{i j} p_{k l}\right) / E(W)
$$

Note that $\Delta_{W}$ may be negative, but $\Delta_{W}+R$ is positive.

In our case, $\sum p_{i j}^{2}=M p^{2}, \Sigma^{\prime} p_{i j} p_{k l}=2 M(n-2) p^{2}, E(W)=M p$, and hence $R$ reduces to

$$
R=2(2 n-3) p<4 n p \text {. }
$$

Hence the theorem is proved.

As a consequence of Theorem 1 , the distribution of $W$ can be approximated by a Poisson distribution if $\Delta_{W}$ and $n p$ are both close enough to zero.

Now assume that there is a $\lambda>0$ such that

$$
E(W) \rightarrow \lambda ; \quad \operatorname{Var}(W) \rightarrow \lambda \text { as } n \text { goes to infinity. }
$$

As seen from (1) this requires that $p$ goes to zero as $1 / n^{2}$ and $c$ goes to zero faster than $1 / n^{3}$ as $n \rightarrow \infty$. Condition (C) is equivalent to

$$
\left(n^{2} / 2\right) P\left(I_{12}=1\right) \rightarrow \lambda ; \quad n^{3} P\left(I_{12}=I_{13}=1\right) \rightarrow 0 .
$$


As a consequence of $(\mathrm{C})$, or $\left(\mathrm{C}^{\prime}\right)$, we have $\Delta_{W} \rightarrow 0$ and $n p \rightarrow 0$ as $n \rightarrow \infty$. Hence Theorem 1 yields the following.

Corollary. If Condition (C), or Condition $\left(\mathrm{C}^{\prime}\right)$, holds then $W$ has, in the limit, a Poisson distribution with mean $\lambda$.

\section{Examples}

Example 1. Uniform distribution over the integers 1 to $N$. Let $\left\{A_{1}, A_{2}, \cdots, A_{n}\right\}$ be a random sample of $n$ values from a uniform distribution over the integers $1,2, \cdots, N$. We find successively

Since

$$
\begin{aligned}
p & =E\left(I_{12}\right)=P\left(I_{12}=1\right)=1 / N, \\
E\left(I_{12} I_{13}\right) & =P\left(I_{12}=I_{13}=1\right)=1 / N^{2}, \\
c & =\operatorname{Cov}\left(I_{12}, I_{13}\right)=0, \\
E(W) & =M / N, \\
\operatorname{Var}(W) & =(M / N)(1-1 / N) .
\end{aligned}
$$

$$
\Delta_{W}=-1 / N ; \quad n p=n / N
$$

we conclude from Theorem 1 that the distribution of $W$ has, approximately, a Poisson distribution with mean $M / N$ if $N$ is large and $n / N$ is small. Further, we infer from the Corollary that, when $n$ and $N$ go to infinity in such a way that $M / N \rightarrow \lambda$, then $W$ has, in the limit, a Poisson distribution with mean $\lambda$.

For example, consider 200 random numbers from the set $0000,0001, \cdots, 9999$. Then $n=200, N=10^{4}$ and so $E(W)=\left(\begin{array}{c}200 \\ 2\end{array}\right) / 10^{4}=2 \cdot 0$. As $N$ is large and $n / N=0.02$ is small, we may expect that the distribution of $W$ can be approximated by a Poisson distribution with mean 2.0. In fact, we have from Theorem 1 that $d(W, Z)<0 \cdot 07$, where $Z \sim$ Poisson $(2 \cdot 0)$.

We can also formulate a special case of Example 1 as a birthday problem. Consider the birthdays of $n$ persons. Two persons are said to be similar if they have the same birthday. Assuming the $N=365$ days equally likely as a birthday, the number $W$ of similar pairs among the $n$ persons has mean $\left(\begin{array}{c}n \\ 2\end{array}\right) / 365$. If $n / 365$ is small, it follows from Theorem 1 that the distribution of $W$ can be approximated by a Poisson distribution. For example, take $n=23$ which is an often quoted value since $P(W \geqq 1)$ is then slightly greater than $1 / 2$ (in fact, it is equal to 0.5073 ). Then $E(W)=0.69$ and Theorem 1 yields $d(W, Z)<0 \cdot 12$; this is not very informative. Hence we cannot decide in this way whether the Poisson approximation is good or not. Note, however, that when $n=23$ the Poisson approximation yields $P(Z \geqq 1)=0 \cdot 5000$ which is a good approximation of the correct value 0.5073; cf. Schwartz (1988). Remember that the variational distance gives an upper bound for the error of the Poisson approximation of the probability of any event $\{W \in B\}$.

Example 2. Non-uniform distribution. Consider again a distribution over 1 to $N$, where $k$ now occurs with probability $p_{k}, k=1,2, \cdots, N, \sum p_{k}=1$. Setting $p=\sum p_{k}^{2}, r=\sum p_{k}^{3}$, we find

$$
\begin{aligned}
E(W) & =M p ; \quad \operatorname{Var}(W)=M p(1-p)+2 M(n-2)\left(r-p^{2}\right), \\
\Delta_{W} & =2(n-2)(r / p-p)-p .
\end{aligned}
$$

By Theorem 1 we can use the Poisson approximation if $\Delta_{W}$ and $n p$ are small. Condition $\left(C^{\prime}\right)$ becomes in this case $\left(n^{2} / 2\right) p \rightarrow \lambda, n^{3} r \rightarrow 0$. If these limiting relations are satisfied, it follows from the Corollary that $W$ has, in the limit, a Poisson distribution with mean $\lambda$.

Example 3. DNA breakages. In Cowan et al. (1987), a model for studying damage to circular DNA is studied. The mathematical model can be described as follows. Let a circle have a circumference of length 1 . On the circumference $n$ points are independently plotted using a uniform distribution. Each point is marked 0 or 1 , independently by flipping a fair 
coin. If two points with different marks are too close, the circle breaks. We seek the probability of this event.

To be more precise, suppose that elements $A_{i}=\left(P_{i}, X_{i}, U_{i}\right)$ are generated in the following way: the points, $P_{1}, \cdots, P_{n}$, are taken from a uniform distribution on the circumference, the critical distances, $X_{1}, \cdots, X_{n}$, are i.i.d. random variables and the marks, $U_{1}, \cdots, U_{n}$, are Bernoulli $\left(\frac{1}{2}\right)$ random variables. The $P$ 's, $X$ 's and $U$ 's are all independent. Let $D_{i j}$ be the arc-length distance between $P_{i}$ and $P_{j}$ and define $I_{i j}=I$ if $U_{i}$ and $U_{j}$ are different and $D_{i j}<\min \left(X_{i}, X_{j}\right)$, and $I_{i j}=0$ otherwise. When $I_{i j}=1$ the elements $A_{i}$ and $A_{j}$ are said to form a similar pair. The event, 'no breakage occurs', is equivalent to the event $W=\sum_{i<j} I_{i j}=0$. Thus we have $P($ no breakage $)=P(W=0)$.

The indicators $I_{i j}$ have the structure described in Section 1. Conditional on $X_{1}, X_{2}$ the event $I_{12}=1$ happens with probability

$$
\left(2 \min \left(X_{1}, X_{2}\right)\right) \cdot(1 / 2)=\min \left(X_{1}, X_{2}\right) .
$$

Further, the event $I_{12}=I_{13}=1$ occurs with probability

Hence we have to take

$$
\min \left(X_{1}, X_{2}\right) \min \left(X_{1}, X_{3}\right) \text {. }
$$

$$
\begin{aligned}
& p=E\left[\min \left(X_{1}, X_{2}\right)\right] \\
& c=\operatorname{Cov}\left(\min \left(X_{1}, X_{2}\right), \min \left(X_{1}, X_{3}\right)\right]
\end{aligned}
$$

in (1).

If $\Delta_{W}$ given by (2) and $n p$ are small, Theorem 1 shows that the distribution of $W$ can be approximated by a Poisson distribution. As a consequence we obtain

$$
P(W=0) \approx \exp \left[-\left(\begin{array}{l}
n \\
2
\end{array}\right) E\left(\min \left(X_{1}, X_{2}\right)\right]\right. \text {. }
$$

For example, if the $X$ 's are uniformly distributed over the interval $(0, b)$, it is found that $E\left(X_{1}\right)=b / 2$ and $E\left[\min \left(X_{1}, X_{2}\right)\right]=b / 3$. Also it is seen after some calculation that $c=b^{2} / 45$. Inserting these values in the inequality of Theorem 1 we can judge whether the variational distance is small enough to allow the Poisson distribution to be used. If this is possible, we conclude, finally, that

$$
P(W=0) \approx \exp \left[-\left(\begin{array}{l}
n \\
2
\end{array}\right) \frac{b}{3}\right]
$$

This is then, approximately, the probability of no breakage.

For further results on the case $X_{i} \equiv b / 2$, constant, see Cowan et al. (1990) and Holst (1989).

\section{References}

Barbour, A. D. and Eagleson, G. K. (1983). Poisson approximation for some statistics based on exchangeable trials. Adv. Appl. Prob. 15, 585-600.

Barbour, A. D. AND EAgleson, G. K. (1984) Poisson convergence for dissociated statistics. J. $R$. Statist. Soc. B 46, 397-402.

Cowan, R., Collis, C. M. AND CRIGG, G. W. (1987) Breakage of double-stranded DNA due to single-stranded nicking. J. Theor. Biol. 127, 229-246.

Cowan, R., Culpin, D. AND Gates, D. (1990) Asymptotic results for a problem of DNA breakage.

J. Appl. Prob. 27 (2).

HOLST, L. (1989) A circle covering problem and DNA breakage. Statist. Prob. Lett. 8 (2).

Schwartz, W. (1988) Approximating the birthday problem. Amer. Statistician 42, 195-196. 